\title{
Daily Budget Optimization Based on the Newsboy Model for Advertisers in the SSA Markets
}

\author{
Linjing Li $^{1}{ }^{1}$ Daniel Zeng ${ }^{1,2}$ Yanwu Yang ${ }^{1}$ \\ ${ }^{1}$ Key Laboratory of Complex Systems and Intelligence Science \\ Institute of Automation, Chinese Academy of Sciences \\ Beijing 100190, P. R. China \\ ${ }^{2}$ Department of Management Information Systems, The University of Arizona \\ Tucson AZ 85721, USA
}

\begin{abstract}
This paper provides a method for planing the optimal daily budget in the online sponsored search advertising market, which is now the most important marketing place. The proposed method is mainly based the well-known newsboy model, which has been widely applied in single period inventory management settings. We model this budget optimization question as a decision problem under a situation where both the future price and demand are uncertain. We also indicate that advertisers will invest more money in the SSA market if the potential profit of clicks in this market is relatively larger than the financial cost of money.
\end{abstract}

Keywords: Newsboy, Budget, SSA

\section{Introduction}

Sponsored search advertising (SSA) is the most prevailing and important online advertising instrument provided by search engines. According to a secret document revealed by AdAge ${ }^{1}$, in June

\footnotetext{
${ }^{1}$ http://tech.fortune.cnn.com/2010/09/ 06/adage-reveals-secret-google-adwords-b ig-spenders/
}

this year, the top three big spenders in Google AdWords system have spent $\$ 8.08 \mathrm{M}, \$ 6.67 \mathrm{M}$, and $\$ 5.95 \mathrm{M}$ for advertising, respectively.

In the SSA market, advertisers submit advertisements to search engine and buy some keywords that related to their advertisements. If one of these keywords matching the query of a search engine user, the search engine will show one of these advertisements along with the standard search results. If the user clicks on the link, then the advertiser should pay a certain fee to the search engine for bringing this potential customer to its website. For more detailed information on SSA, please refer to $[1,2]$.

Keywords used in SSA market are sold by auctions. Two major auction formats have been used in practice: the generalized first-price and the generalized second-price mechanisms [1-4]. In this paper, we do not want to discuss the details of specific mechanisms. Readers please refer to [4-10] for the discussions of equilibrium in keywords auction mechanisms; and [11-17] for the design and comparison of new auction mechanisms.

In this paper, we propose a daily 
budget optimization method for the advertisers based on the well-known single period newsboy (or newsvendor) model [18-20]. First, we extend the classical newsboy model to address individual decision problem in a situation where both the future price and future demand are uncertain. Then, we use this extended model in the SSA market setting to obtain the optimal daily budget. Based on this model, we conclude that the advertisers would prefer to spend more money in the SSA market, if the potential profit of clicks is relatively higher than the cost of money. Otherwise, they would like to decline the money spent in this market.

The remainder of this paper is organized as follows. We illustrate how to formulate the budget optimization problem as a newsboy model in Section 2. Section 3 provides some examples and further discussions on our model. Finally, Section 4 summarizes our concluding remarks and lists some future research directions.

\section{Daily Budget Optimization}

In this paper, we consider a macroscope setting that an advertiser faced in the SSA market, in which we do not care details of the specific auction mechanisms used by search engines; and treat the daily budget optimization as an individual decision problem under uncertainties. However, it is also possible to model this problem as a game as have done in $[21,22]$, we will try this model in our future work.

\subsection{Newsboy Model}

Let $C$ be the daily clicks an advertisement can received, it is a random variable defined on $\left[c_{m}, c_{M}\right]$ with density $g(c)$ and distribution $G(c)$, where $c_{m}$ and $c_{M}$ are the minimum and maximum of daily clicks, respectively. Similarly, let $P$ be the average price of a click on this advertisement, it is a random variable defined on $\left[p_{m}, p_{M}\right]$ with density $h(p)$ and distribution $H(p)$, where $p_{m}$ and $p_{M}$ are the minimum and maximum of price, respectively. In general, these two random variables are correlated, so we denote their joint density and distribution as $f(c, p)$ and $F(c, p)$; and let $\Omega=\left[c_{m}, c_{M}\right] \times\left[p_{m}, p_{M}\right]$ represent the domain of these two random variables.

We formulate the daily budget allocation problem as a single-period newsboy model [18-20]. To do so, let $q$ be the daily budget an advertiser submitted. If the actual daily expenditure $c p$ is less than the pre-submitted budget $q$, the unspent money $q-c p$ will cause an overage cost $\gamma_{o}(q-c p)$, where $\gamma_{o}$ is the unit cost of overage. However, if the advertisers do not put enough budget into the SSA system, the search engines will shut off their advertisements. Thus, advertisers may lose some potential profit in this situation. Let $\gamma_{s}$ be the unit potential profit of shortage, then the total shortage loss can be represented as $\gamma_{s}(c p-q)$. In general, $\gamma_{o}$ can be treated as the risk-free interest, whereas $\gamma_{s}$ is the average expected rate of ROI (return-on-investment) of the SSA market.

Using these symbols, we can denote the total loss of submitting daily budget $q$ as

$$
\begin{aligned}
L(q) & =\iint_{\Omega_{\mathrm{s}}} \gamma_{s}(c p-q) f(c, p) \mathrm{d} c \mathrm{~d} p \\
& +\iint_{\Omega_{\mathrm{o}}} \gamma_{o}(q-c p) f(c, p) \mathrm{d} c \mathrm{~d} p
\end{aligned}
$$

where the first term represents the total loss of shortage and the second term is the total overage loss; $\Omega_{\mathrm{S}}$ is the re- 
gion of shortage, whereas $\Omega_{0}$ is the region of shortage, such that $\Omega=\Omega_{\mathrm{s}} \cup \Omega_{\mathrm{O}}$. Both $\Omega_{\mathrm{s}}$ and $\Omega_{\mathrm{o}}$ can be empty, to reflect the situations of absolute shortage when the budget is very small, i.e., $q \leq c_{m} p_{m}$, and absolute overage when the budget is extremely high, i.e., $q \geq c_{M} p_{M}$. Figure 1 shows some simple examples.

\subsection{Budget Optimization}

In the following, we discuss how to find the optimal daily budget $q^{\star}$, i.e., the one minimizes the total loss $L(q)$ given by equation (1). To form a complete analysis, we must consider the following three situations: $c_{m} p_{M} \leq c_{M} p_{m}$, $c_{m} p_{M}=c_{M} p_{m}$, and $c_{m} p_{M} \geq c_{M} p_{m}$. However, all three situations use the same analysis method, thus, we only present the analysis of situation where $c_{m} p_{M} \leq c_{M} p_{m}$ in the rest of this section. According to figure 1, we still have to analyze five different cases.

Case 1: $q \leq c_{m} p_{m}$. Obviously, in this case, $q$ is absolutely shortage, thus $\Omega_{\mathrm{o}}=\emptyset$ and $\Omega_{\mathrm{s}}=\Omega$. The total loss $L(q)$ is then

$$
\begin{aligned}
L(q) & =\iint_{\Omega} \gamma_{s}(c p-q) f(c, p) \mathrm{d} c \mathrm{~d} p \\
& =\gamma_{s} \iint_{\Omega} c p f(c, p) \mathrm{d} c \mathrm{~d} p-\gamma_{s} q .
\end{aligned}
$$

Note that $L^{\prime}(q)=-\gamma_{s}<0$, thus we conclude that the optimal budget in this case is $q_{1}^{\star}=c_{m} p_{m}$, and the minimal total loss is $L\left(q_{1}^{\star}\right)=$ $\gamma_{s} \iint_{\Omega} c p f(c, p) \mathrm{d} c \mathrm{~d} p-\gamma_{s} c_{m} p_{m}$.

Case 2: $q \geq c_{M} p_{M}$. In this case, $q$ is absolutely overage, thus $\Omega_{\mathrm{s}}=\emptyset$ and $\Omega_{\mathrm{o}}=\Omega$. The total loss $L(q)$ is then

$$
\begin{aligned}
L(q) & =\iint_{\Omega} \gamma_{o}(q-c p) f(c, p) \mathrm{d} c \mathrm{~d} p \\
& =\gamma_{o} q-\gamma_{o} \iint_{\Omega} c p f(c, p) \mathrm{d} c \mathrm{~d} p .
\end{aligned}
$$

Note that $L^{\prime}(q)=\gamma_{o}>0$, thus we conclude that the optimal budget in this case is $q_{2}^{\star}=c_{M} p_{M}$, and the minimal total loss is $L\left(q_{2}^{\star}\right)=\gamma_{o} c_{M} p_{M}-$ $\gamma_{o} \iint_{\Omega} c p f(c, p) \mathrm{d} c \mathrm{~d} p$.

To discuss the rest three cases, we need the following lemma on the derivative of function defined by a double definite integral.

Lemma 2.1 Let $k(t), \alpha \leq t \leq \beta$, be a function defined by the following double definite integral

$$
k(t)=\int_{\varphi_{l}(t)}^{\varphi_{u}(t)} \mathrm{d} x \int_{\theta_{l}(x, t)}^{\theta_{u}(x, t)} f(x, y, t) \mathrm{d} y,
$$

where both $\varphi_{l}(t)$ and $\varphi_{u}(t)$ are differentiable; both $\theta_{l}(x, t)$ and $\theta_{u}(x, t)$ are differentiable with respect to $t ; f(x, y, t)$ is differentiable with respect to $t$ and continuous on region $D=\left\{(x, y) \mid \varphi_{l}(t) \leq\right.$ $\left.x \leq \varphi_{u}(t), \theta_{l}(x, t) \leq y \leq \theta_{u}(x, t)\right\}$. Then, $k(t)$ is differentiable and its derivative can be represented as follows

$$
\begin{aligned}
k^{\prime}(t) & =\varphi_{u}^{\prime} \int_{\theta_{l}\left(\varphi_{u}, t\right)}^{\theta_{u}\left(\varphi_{u}, t\right)} f\left(\varphi_{u}(t), y, t\right) \mathrm{d} y \\
& -\varphi_{l}^{\prime} \int_{\theta_{l}\left(\varphi_{l}, t\right)}^{\theta_{u}\left(\varphi_{l}, t\right)} f\left(\varphi_{l}(t), y, t\right) \mathrm{d} y \\
& +\int_{\varphi_{l}}^{\varphi_{u}} \frac{\partial \theta_{u}(x, t)}{\partial t} f\left(x, \theta_{u}(x, t), t\right) \mathrm{d} x \\
& -\int_{\varphi_{l}}^{\varphi_{u}} \frac{\partial \theta_{l}(x, t)}{\partial t} f\left(x, \theta_{l}(x, t), t\right) \mathrm{d} x \\
& +\iint_{D} \frac{\partial f(x, y, t)}{\partial t} \mathrm{~d} x \mathrm{~d} y .
\end{aligned}
$$

The proof of this lemma is a straightforward application of the definition of limit and the integral mean value theorem, and very cumbersome, so we omit the proof details.

Case 3: $c_{M} p_{m} \leq q \leq c_{M} p_{M}$. In this case, $\Omega_{\mathrm{s}}=\left\{(c, p) \mid \frac{q}{p_{M}} \leq c \leq\right.$ $\left.c_{M}, \quad \frac{q}{c} \leq p \leq p_{M}\right\}$, see figure $1(\mathrm{~d})$; and $\Omega_{\mathrm{o}}=\Omega_{\mathrm{o}}^{\mathrm{I}} \cup \Omega^{\mathrm{I}}$, where $\Omega_{\mathrm{o}}^{\mathrm{I}}=\left[c_{m}, \frac{q}{p_{M}}\right] \times$ $\left[p_{m}, p_{M}\right]$ and $\Omega_{\mathrm{o}}^{\mathbb{I}}=\left\{(c, p) \mid \frac{q}{p_{M}} \leq c \leq\right.$ 


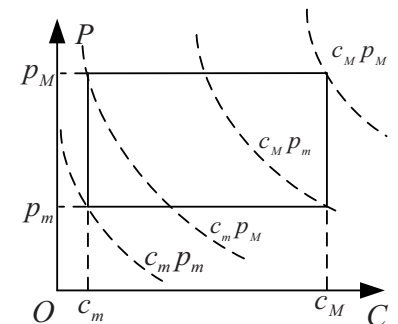

(a) Critical value of $q$

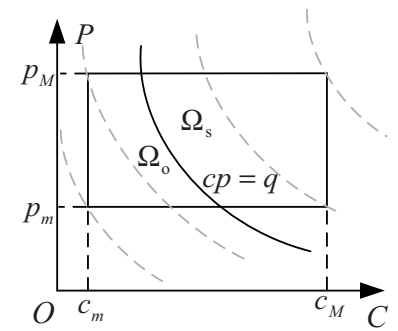

(c) $c_{m} p_{M} \leq q \leq c_{M} p_{m}$

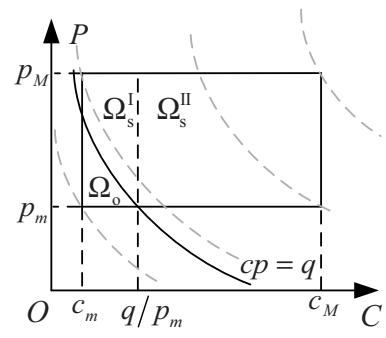

(b) $c_{m} p_{m} \leq q \leq c_{m} p_{M}$

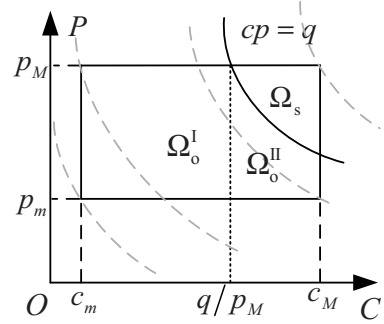

(d) $c_{M} p_{m} \leq q \leq c_{M} p_{M}$

Fig. 1: Shortage $\left(\Omega_{\mathrm{s}}\right)$ and overage $\left(\Omega_{\mathrm{o}}\right)$ regions in case $c_{m} p_{M}<c_{M} p_{m}$.

$\left.c_{M}, p_{m} \leq p \leq \frac{q}{c}\right\}$. The total loss $L(q)$ can then be represented as

$$
\begin{aligned}
L(q) & =\int_{\frac{q}{p_{M}}}^{c_{M}} \mathrm{~d} c \int_{\frac{q}{c}}^{p_{M}} \gamma_{s}(c p-q) f(c, p) \mathrm{d} p \\
& +\int_{c_{m}}^{\frac{q}{p_{M}}} \mathrm{~d} c \int_{p_{m}}^{p_{M}} \gamma_{o}(q-c p) f(c, p) \mathrm{d} p \\
& +\int_{\frac{q}{p_{M}}}^{c_{M}} \mathrm{~d} c \int_{p_{m}}^{\frac{q}{c}} \gamma_{o}(q-c p) f(c, p) \mathrm{d} p .
\end{aligned}
$$

By using the above lemma 2.1, we obtain the derivative of the total loss $L(q)$ as follows

$$
\begin{aligned}
L^{\prime}(q) & =\iint_{\Omega_{\mathrm{o}}^{\mathrm{I}}} \gamma_{o} f(c, p) \mathrm{d} c \mathrm{~d} p \\
& +\iint_{\Omega_{\mathrm{o}}^{\mathrm{II}}} \gamma_{o} f(c, p) \mathrm{d} c \mathrm{~d} p \\
& -\iint_{\Omega_{\mathrm{s}}} \gamma_{s} f(c, p) \mathrm{d} c \mathrm{~d} p .
\end{aligned}
$$

To obtain the optimal daily budget $q^{\star}$, we simply let $L^{\prime}\left(q^{\star}\right)=0$, and obtain the following condition

$$
\begin{gathered}
F\left(\frac{q^{\star}}{p_{M}}, p_{M}\right)+\int_{\frac{q^{\star}}{p_{M}}}^{c_{M}} \mathrm{~d} c \int_{p_{m}}^{\frac{q^{\star}}{c}} f(c, p) \mathrm{d} p \\
=\frac{\gamma_{s}}{\gamma_{s}+\gamma_{o}} .
\end{gathered}
$$

In general, the solution of the above equation is depending on the joint density function $f(c, p)$ (or joint distribution function $F(c, p)$ ), and does not have a explicit expression. Note that, equation (2) and (3) have the same form as those in the classical newsboy model [18-20]. We will provide further discussions on this in the next section.

Let $\mathcal{S}$ be the solution set of the above equation (3). To get the optimal budget in this case, we still need to consider the boundary value $q=c_{M} p_{m}$ and $q=$ $c_{M} p_{M}$. Define $\hat{\mathcal{S}}=\mathcal{S} \cup\left\{c_{M} p_{m}, c_{M} p_{M}\right\}$, and the set of optimal budgets in this 
case can then be represented as

$$
\mathcal{Q}_{1}=\left\{q^{\star} \mid q^{\star}=\underset{q \in \hat{\mathcal{S}}}{\arg \min } L(q)\right\} .
$$

Case 4 and 5: Apply the same analysis to the rest two cases: $c_{m} p_{m} \leq q \leq$ $c_{m} p_{M}$ and $c_{m} p_{M} \leq q \leq c_{M} p_{m}$, we can obtain another two candidate sets for the optimal budget: $\mathcal{Q}_{2}$ and $\mathcal{Q}_{3}$, respectively. The corresponding shortage and overage regions are indicated by figure $1(\mathrm{~b})$ and $1(\mathrm{c})$. To obtain the final solution, define

$$
\mathcal{Q}=\mathcal{Q}_{1} \cup \mathcal{Q}_{2} \cup \mathcal{Q}_{3} .
$$

Then, the optimal budget $q^{\star}$ can be represented as

$$
q^{\star}=\underset{q \in \mathcal{Q}}{\arg \min } L(q) .
$$

And the minimal total loss $L\left(q^{\star}\right)$ is

$$
L\left(q^{\star}\right)=\min _{q \in \mathcal{Q}} L(q) .
$$

When there exists multiple optimums, we can safely choose the minimal one to avoid additional risk.

\section{Examples and Discussions}

In this section, we analysis two special cases and a numeric example to illustrate the above analysis method.

First, consider the case that random variables $C$ and $P$ are independent, i.e., $f(c, p)=g(c) h(p)$, or $F(c, p)=$ $G(c) H(p)$, then equation (3) in the past section can be simplified as

$$
\begin{gathered}
G\left(\frac{q^{\star}}{p_{M}}\right)+\int_{\frac{q^{\star}}{p_{M}}}^{c_{M}} H\left(\frac{q^{\star}}{c}\right) g(c) \mathrm{d} c \\
=\frac{\gamma_{s}}{\gamma_{s}+\gamma_{o}} .
\end{gathered}
$$

Second, assume $F(c, p)$ subject to 2dimensional uniform distribution, i.e.,

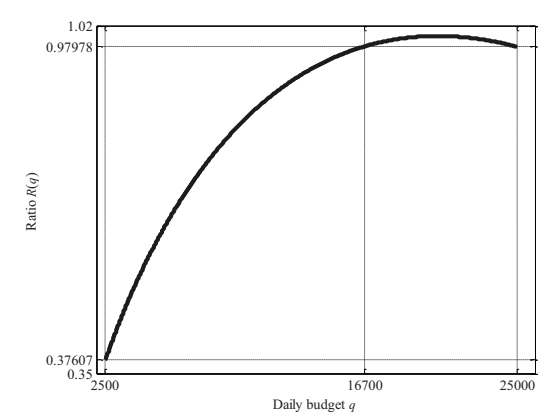

Fig. 2: $R(q)$ vs. $q$.

$f(c, p) \equiv \frac{1}{q_{m}}$, where $q_{m}=\left(c_{M}-\right.$ $\left.c_{m}\right)\left(p_{M}-p_{m}\right)$. Obviously, in this case, random variable $C$ and $P$ are independent. Substitute the above density and distribution functions into equation (4), it can be simplified as

$$
\frac{q^{\star}}{q_{m}}\left[1+\ln \left(\frac{q_{m}}{q^{\star}}\right)\right]=\frac{\gamma_{s}}{\gamma_{s}+\gamma_{o}} .
$$

Consider a numeric example, where $c_{m}=200, c_{M}=2500, p_{m}=1$, and $p_{M}=10$. In this example, $c_{m} p_{M}=$ $2000 \leq c_{M} p_{m}=2500$. Define $R(q)=$ $\frac{q}{q_{m}}\left[1+\ln \left(\frac{q_{m}}{q}\right)\right]$, Figure 2 shows this function in range $2500 \leq q \leq 25000$.

Define $\beta=\frac{\gamma_{s}}{\gamma_{s}+\gamma_{o}}$ be the critical ratio, which reflects the relative importance of shortage and overage. As the same as the classical newsboy model [18-20], $\beta$ is a increasing function with respect to the first parameter $\gamma_{s}$, and a decreasing function with respect to the second parameter $\gamma_{o}$.

Combine with figure 2 , we find that there exists a threshold $\beta_{0}$ (about 0.3761 in the above numeric example) for the critical ratio, when $\beta<\beta_{0}$, the optimal budget does exist in this region. This is mainly because that this kind of advertisers are more worried about the overage cost, as a result, they put a relatively low budget into the SSA market. 
When the actual critical ratio $\beta$ is larger than $\beta_{0}$, we find that the daily budget is a increasing function with respect to $\beta$, until it reaches the maximal critical ratio $\beta=1$. This indicates that if advertisers take more considerations to the potential profit, they will prefer to put more budget into this market.

However, as the above figure shows, there exists another key-point $\beta_{c}$ (about 0.9798 in our example) about the critical ratio. We can find a unique optimum when the actual critical ratio is less than this critical value, whereas there exists two optimums. In this situation, we can safely choose the minimal one to avoid additional risk.

\section{Conclusion}

This paper provides a daily budget optimization method for the advertisers in the SSA market. The proposed method is based on the well-known single period newsboy model. We extended the classical model to be able to address individual decision problem in which both the future price and demand are uncertain, thus it can be used to obtain the optimal daily budget in a SSA market.

According to our analysis, if the advertisers were interested in obtaining potential profit, they would prefer to spend more budget in the SSA market. Otherwise, if they were worried about the financial cost of money, they would like to decline the daily budget.

In our ongoing work, we plan to extend this single period model to address dynamic budget optimization problem which requires allocating budget across a long period, such as a month, a quarter, or even a whole year. We are also dedicating to model this problem as a game theoretical, or competitive, newsboy model.

\section{Acknowledgements}

This work was supported in part by the National Natural Science Foundation of China under grant 60875049 , 60921061, 70890084, and 71071152; by the Chinese Ministry of Science and Technology under grant 2006AA010106; and by the Chinese Academy of Sciences under grant 2F07C01.

\section{References}

[1] Bernard J. Jansen and Tracy Mullen. Sponsored search: an overview of the concept, history, and technology. International Journal of Electronic Business, 6(2):114-131, 2008.

[2] S. Muthukrishnan. Internet ad auctions: Insights and directions. In Proceedings of the 35th international colloquium on $A u$ tomata, Languages and Programming, Part I, volume 5125, pages 14-23, 2008.

[3] Benjamin Edelman and Michael Ostrovsky. Strategic bidder behavior in sponsored search auctions. Decision Support Systems, 43(1):192 - 198, 2007.

[4] Benjamin Edelman, Michael Ostrovsky, and Michael Schwarz. Internet advertising and the generalized second-price auction: Selling billions of dollars worth of keywords. American Economic Review, 97(1):242-259, 2007.

[5] Hal R. Varian. Position auctions. International Journal of Industrial Organization, 25(6):11631178, 2007.

[6] Matthew Cary, Aparna Das, Ben Edelman, Ioannis Giotis, Kurtis Heimerl, Anna R. Karlin, Claire Mathieu, and Michael Schwarz. 
Greedy bidding strategies for keyword auctions. In Proceedings of the 8th ACM conference on Electronic commerce, pages 262-271, New York, NY, USA, 2007. ACM.

[7] Paul Milgrom. Simplified mechanisms with an application to sponsored-search auctions. Games and Economic Behavior, In Press, Corrected Proof, 2008.

[8] Hal R. Varian. Online ad auctions. American Economic Review, 99(2):430-434, 2009.

[9] Linjing Li, Daniel Zeng, and Feiyue Wang. Equilibrium bidding strategy for GSP keyword auctions. In Proceedings of The 19th Workshop on Information Technologies and Systems, pages 109 114, Phoenix, Arizona, USA, Dec. 14-15 2009.

[10] Linjing Li and Daniel Zeng. Refinement of symmetrical nash equilibrium for generalized second-price mechanism in sponsored search advertising. In Proceedings of the 2010 IEEE International Conference on Service Operations and Logistics, and Informatics (SOLI 10), pages 457-462, Qingdao, Shandong, China, July 2010.

[11] Alvin E. Roth and Marilda A. Oliveira Sotomayor. TwoSided Matching: A Study in Game-Theoretic Modeling and Analysis. Cambridge University Press, June 1992.

[12] G. Iyengar and A. Kumar. Characterizing optimal keyword auctions. In Proceedings of the 2nd Workshop on Sponsored Search Auctions, Michigan, 2006.

[13] G. Aggarwal, A. Goel, and R. Motwani. Truthful auctions for pricing search keywords. In Proceedings of the \%th ACM Con- ference on Electronic Commerce, pages 1-7, 2006.

[14] D. Garg, Y. Narahari, and S. S. Reddy. Design of an optimal auction for sponsored search auctions. In $C E C$-EEE'07, 2007.

[15] N. Lambert and Y. Shoham. Asymptotically optimal repeated auctions for sponsored search. In ICEC'07, Minnesota, 2007.

[16] D. Bergemanny and J. Valimakiz. Dynamic efficient auctions. In Third Workshop on Sponsored Search Auctions, Banff, Canada, 2007.

[17] Linjing Li, Daniel Zeng, and Paulo Goes. How good is the generalized second-price mechanism? In Proceedings of the 20th Workshop on Information Technologies and Systems (WITS 2010), St. Louis, Missouri, USA, December 2010.

[18] Kenneth J. Arrow, Theodore Harris, and Jacob Marschak. Optimal inventory policy. Econometrica, 19:250-272, 1951.

[19] Kenneth J. Arrow, S. Karlin, and H. Scarf. Studies in the mathematical theory of inventory and production. Stanford University Press, Stanford, CA, 1958.

[20] E. L. Porteus. Stochastic inventory theory. In D. P. Heyman and M. J. Sobel, editors, Handbooks in $O R$ and $M S$, volume 2, pages 605652. Elsevier Science Publishers, 1990.

[21] Steven A. Lippman and Kevin F. McCardle. The competitive newsboy. Operations Research, 45(1):54-65, 1997.

[22] M. Parlar. Game theoretic analysis of the substitutable product inventory problem with random demands. Naval Res. Logist, 35:397409, 1988. 